\title{
Hochdosiertes Biotin wirkt bei progressiver MS
}

Fragestellung: Ist hochdosiertes Biotin bei den chronisch progredienten, nicht entzündlich aktiven Verlaufsformen der Multiplen Sklerose (MS) wirksam?

Hintergrund: Basierend auf dem Vorhandensein von entzündlicher Krankheitsaktivität kann die primär progrediente (PP) und sekundär chronisch progrediente (SP) Verlaufsform der MS in eine entzündliche und nicht entzündliche aktive Form gemäß der im Jahre 2013 revidierten Lublin-Klassifikation unterteilt werden [1]. Biotin ist das Coenzym für vier Carboxylasen des Menschen. Angenommen wird, dass die hochdosierte Gabe von Biotin zur Aktivierung dieser Carboxylasen beiträgt. Postuliert werden die Aktivierung der Fettsäuresynthese und die vermehrte Bereitstellung von intrazellulären Metaboliten, wodurch degenerative Mechanismen verhindert werden und die Regeneration von Myelin gefördert wird.

Tourbah A, Lebrun-Frenay C, Edan G et al. MD1003 (highdose biotin) for the treatment of progressive multiple sclerosis: A randomised, double-blind, placebo-controlled study. Multiple Sclerosis J 2016; 22: 1719-31
Patienten und Methodik: Die doppelblinde, randomisierte und placebokontrollierte Studie schloss Probanden mit PPMS und SPMS mit einm Baseline-Wert in der EDSS (Expanded Disability Status Scale) von 4,5-7,0 ein, bei denen in den letzten zwei Jahren eine gesicherte klinische Progredienz vorlag. Im Anschluss an die zwölfmonatige Studie erfolgte eine zwölfmonatige Open-label-Extensionsphase. Biotin wurde oral in einer Dosis von $3 \times 100 \mathrm{mg}$ täglich verabreicht. Primärer Outcome war der Anteil an Probanden, bei denen im Monat 12 eine gesicherte Besserung in der EDSS und/oder im T25W (Timed 25-Foot Walk) im Vergleich zum besten Wert bei Randomisierung beziehungsweise Baseline vorlag.

Ergebnisse: 154 Probanden wurden randomisiert (Placebo $\mathrm{n}=$ 51, Biotin $n=103$ ). Neun Probanden im Placebo- und zwölf im Behandlungsarm beendeten die Studie vorzeitig. Insgesamt erreichten 13 Probanden (12,6\%) in der Behandlungsgruppe den primären Outcome. In der Extensionsphase kamen fünf weitere Probanden hinzu (zwei aus der Behandlungs- und drei aus der ursprünglichen Placebogruppe). Hinsichtlich sekundärer Outcomes zeigte sich bei 13,6\% der Probanden im Placebo- und 4,2 \% im Behandlungsarm eine EDSS-Verschlechterung bei Studienende (Monat 12). Wichtige Nebenwirkungen der Biotin-Behandlung waren laborchemische Veränderungen im Sinne einer Hyperthyreose $(n=6)$ und mukokutaner Hautausschlag $(n=1)$.

Schlussfolgerung: Die Autoren folgern, dass Biotin eine effektive und sichere Therapie für MS-Patienten mit progressiver Verlaufsform darstellt.

\section{- Kommentar von Refik Pul und Christoph Kleinschnitz, Essen}

\section{Wirkung auf degenerative Mechanismen nicht bewiesen}

In einer vorausgegangenen, offenen Studie haben die Autoren bei 20 von 23 MS-Patienten eine klinische Besserung durch hochdosiertes Biotin feststellen können [2]. An dieses Ergebnis können sie in der vorliegenden, kontrolliert durchgeführten Studie nicht anknüpfen. Immerhin beobachteten sie nach einem Jahr bei 12,6\% und zum Ende der Extensionsphase bei $13,2 \%$ der Patienten eine klinische Besserung, wobei die primären Outcomes dieser Studie für einen kurzen Zeitraum von nur zwölf Monaten sehr ambitioniert gesteckt wurden. Die Studie weist Schwächen auf, welche die Schlussfolgerung der Autoren in Bezug auf die Wirkung bei nicht entzündlich aktiver MS relativieren. Bei 47 Patienten im Behandlungs- und 23 im Placeboarm erfolgte eine kranielle Kernspintomografie zu Studienbeginn und -ende. Da bei 23,4\% im Behandlungs- und $8,5 \%$ im Placeboarm bildgebend eine Progression vorlag, stellt sich die Frage, ob der beobachtete Effekt nicht das Resultat einer antiinflammatorischen Wirkung war. In diesem Zusammenhang sei noch erwähnt, dass von den 13 MS-Patienten, die einen oder beide primären Outcomes erfüllten, neun einen sekundär und nur vier einen primär progredienten Verlauf hatten. Schwachpunkte der Arbeit sind die Statistik, die auf einfachen, nicht parametrischen Gruppenvergleichen basierte, und dass die Autoren dazu neigen, ihre Ergebnisse insbesondere hinsichtlich der sekundären Outcomes überzuinterpretieren. Auf Basis ihrer Vorarbeit wird die Probandenanzahl begründet, die Autoren bleiben aber eine nachträgliche Poweranalyse schuldig. Die aktuelle Studie rückt ein weiteres Vitamin, neben Vitamin D, in den Fokus der MS-Behandlung. Obgleich sie eine gewisse Wirkung bei MS-Patienten mit chronisch progredienter Verlaufsform aufzeigt, so kann sie die postulierte Wirkung auf die degenerativen Krankheitsmechanismen nicht belegen.
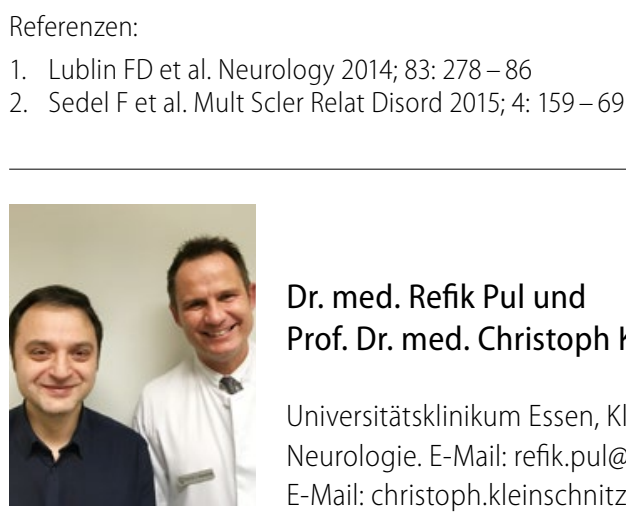

Dr. med. Refik Pul und

Prof. Dr. med. Christoph Kleinschnitz

Universitätsklinikum Essen, Klinik für Neurologie. E-Mail: refik.pul@uk-essen.de E-Mail: christoph.kleinschnitz@uk-essen.de 